\title{
Biphasic dynamics of beta cell mass in a mouse model of congenital hyperinsulinism: implications for type 2 diabetes
}

\author{
Sharona Tornovsky-Babeay ${ }^{1,2} \cdot$ Noa Weinberg-Corem ${ }^{3} \cdot$ Rachel Ben-Haroush Schyr $^{3}$ (D) - Dana Avrahami ${ }^{1,2,3}$ (D) \\ Judith Lavi $^{1,2} \cdot$ Eseye Feleke $^{1,2} \cdot$ Klaus H. Kaestner $^{4}$ (D) Yuval Dor $^{3}$ (D) Benjamin Glaser ${ }^{1,2}$ (D)
}

Received: 9 September 2020 / Accepted: 8 December 2020 / Published online: 9 February 2021

(C) The Author(s), under exclusive licence to Springer-Verlag GmbH, DE part of Springer Nature 2021

\begin{abstract}
Aims/hypothesis Acute hyperglycaemia stimulates pancreatic beta cell proliferation in the mouse whereas chronic hyperglycaemia appears to be toxic. We hypothesise that this toxic effect is mediated by increased beta cell workload, unrelated to hyperglycaemia per se.

Methods To test this hypothesis, we developed a novel mouse model of cell-autonomous increased beta cell glycolytic flux caused by a conditional heterozygous beta cell-specific mutation that activates glucokinase (GCK), mimicking key aspects of the rare human genetic disease GCK-congenital hyperinsulinism.

Results In the mutant mice, we observed random and fasting hypoglycaemia (random 4.5-5.4 mmol/1 and fasting 3.6 mmol/l) that persisted for 15 months. GCK activation led to increased beta cell proliferation as measured by Ki67 expression (2.7\% vs $1.5 \%$, mutant and wild-type (WT), respectively, $p<0.01$ ) that resulted in a $62 \%$ increase in beta cell mass in young mice. However, by 8 months of age, mutant mice developed impaired glucose tolerance, which was associated with decreased absolute beta cell mass from $2.9 \mathrm{mg}$ at $1.5 \mathrm{months}$ to $1.8 \mathrm{mg}$ at 8 months of age, with preservation of individual beta cell function. Impaired glucose tolerance was further exacerbated by a high-fat/high-sucrose diet (AUC $1796 \mathrm{vs} 966 \mathrm{mmol} / \mathrm{l} \times \mathrm{min}$, mutant and WT, respectively, $p<0.05)$. Activation of GCK was associated with an increased DNA damage response and an elevated expression of Chop, suggesting metabolic stress as a contributor to beta cell death.

Conclusions/interpretation We propose that increased workload-driven biphasic beta cell dynamics contribute to decreased beta cell function observed in long-standing congenital hyperinsulinism and type 2 diabetes.
\end{abstract}

Keywords Beta cell · Glucokinase $\cdot$ Glucose intolerance · Glucotoxicity $\cdot$ Hyperglycaemia $\cdot$ Hypoglycaemia $\cdot$ Insulin secretion · Mouse model · Type 2 diabetes

Yuval Dor

yuvald@ekmd.huji.ac.il

Benjamin Glaser

ben.glaser@mail.huji.ac.il

1 Department of Endocrinology and Metabolism, Hadassah Medical Center, Jerusalem, Israel

2 Faculty of Medicine, Hebrew University of Jerusalem, Jerusalem, Israel

3 Department of Developmental Biology and Cancer Research, The Hebrew University-Hadassah Medical School, Jerusalem, Israel

4 Department of Genetics and Institute for Diabetes, Obesity and Metabolism, University of Pennsylvania, Philadelphia, PA, USA

$\begin{array}{ll}\text { Abbreviations } \\ \text { ARC } & \text { Arcuate nucleus } \\ \text { CHI } & \text { Congenital hyperinsulinism } \\ \text { ER } & \text { Endoplasmic reticulum } \\ \text { GCK } & \text { Glucokinase } \\ \text { GCK-CHI } & \text { Glucokinase mutation mediated } \\ & \text { congenital hyperinsulinism } \\ \text { GSIS } & \text { Glucose-stimulated insulin secretion } \\ \text { HFD } & \text { High-fat/high-sugar diet } \\ \text { K ATP } & \text { ATP-sensitive potassium channel } \\ \text { mutGCK } & \text { The GCK gene expressing the } \\ & \text { ins454A activating mutation } \\ \text { qPCR } & \text { Quantitative real-time PCR } \\ \text { TMRE } & \text { Tetramethylrhodamine, ethyl ester } \\ \text { VMH } & \text { Ventro-medial hypothalamus } \\ \text { WT } & \text { Wild-type }\end{array}$




\section{Research in context}

\section{What is already known about this subject?}

- Type 2 diabetes is associated with decreased functional beta cell mass

- Type 2 diabetes is a progressive disease and poor metabolic control is associated with increased rate of progression

- Chronic hyperglycaemia results in beta cell toxicity; however, the mechanisms mediating this are not entirely known

\section{What is the key question?}

- Does increased beta cell workload per se result in long-term toxicity to beta cells?

\section{What are the new findings?}

- We show that while short-term activation of glucokinase in the beta cells results in increased proliferation and beta cell mass, long-term activation results in decreased beta cell mass and glucose intolerance

- This toxicity is mediated by increased oxidative stress and DNA damage

- These results suggest that increased beta cell workload per se has long-term detrimental effects on beta cell survival, even before the advent of hyperglycaemia

\section{How might this impact on clinical practice in the foreseeable future?}

- Intervention aimed at decreasing beta cell workload before the onset of hyperglycaemia may delay the onset of clinical diabetes in the genetically susceptible individual

\section{Introduction}

Type 2 diabetes is characterised by insulin resistance and beta cell dysfunction resulting in hyperglycaemia. While numerous factors including endoplasmic reticulum (ER) stress [1, 2], oxidative stress and glucolipotoxicity [3-5] are suggested to contribute to reduced beta cell function in type 2 diabetes, existing models of diabetes and studies in humans are unable to tease out the relative contributions of various mechanisms to the negative trajectory of net beta cell function. Glucokinase (GCK) (hexokinase-4) phosphorylates glucose to glucose 6-phosphate, the first step of glycolysis, in both beta cells and hepatocytes, acting as the glucose sensor due to its relatively low affinity for glucose. Decreased GCK activity, as seen in MODY2, results in fasting hyperglycaemia, whereas activating GCK mutations result in congenital hyperinsulinism (GCK-CHI), characterised by excessive insulin secretion at low glucose levels leading to potentially lifethreatening hypoglycaemia [6]. We and others have shown that islets of GCK-CHI children are larger and show increased proliferation compared with normal $[7,8]$. These observations, combined with pharmacologic manipulations of GCK activity in mice, highlighted the central role of glycolysis in homeostatic beta cell replication and its potential utility for regenerative therapy in diabetes [9]. This concept suggested the use of small-molecule GCK activators for the treatment of type 2 diabetes [10]. However, most of these molecules failed in clinical trials, raising the possibility of long-term beta cell- toxic effects $[11,12]$. Interestingly, these findings were supported by recent observations that some patients with GCK-CHI ultimately progress to diabetes [13].

Previously, using transgenic mice ectopically expressing a strong activating Gck mutation (Y214C), we identified a molecular pathway that mediates the toxic effects of high glucose flux on beta cells, involving double-strand DNA breaks, activation of P53 and calcium signalling. However, the transgenic system used led to ultra-rapid collapse of beta cells, precluding analysis of long-term beta cell dynamics and putting into question the relevance of this mouse model, given the slow kinetics of human GCK-CHI and type 2 diabetes.

Here, we derived a novel mouse model of GCK-CHI, more faithful to the clinical setting, based on heterozygous expression of a conditional $G c k$ gene replacement allele carrying the severe GCK-activating mutation, ins454A [14, 15], recapitulating the typical long-term mild hypoglycaemia of human GCK-CHI and providing striking insights into the path leading from over-stimulated beta cells to glucose intolerance. Using this new model, we tested the effects of chronic increased beta cell workload on beta cell proliferation, survival rate and function.

\section{Methods}

Derivation of mutGCK mice A mutant mouse model was derived which contains a complex DNA cassette inserted distal to the native Gck exon 9 as described in detail in the 
electronic supplementary material (ESM) (ESM Methods, Fig. 1a). These mice expressed normal GCK in beta cells and all other cell types where GCK is expressed. However, when crossed with mice expressing Cre recombinase (Cre), cells expressing Cre replace the native exon 10 with the mutated exon in situ (Fig. 1a). Mice homozygous for the insert containing ins454A mutant Gck (mutGCK) were crossed either with mice expressing Cre driven by the rat insulin promoter (RIP-Cre) [16] or with mice expressing oestrogendependent Cre driven by the mouse insulin promoter (MIP-

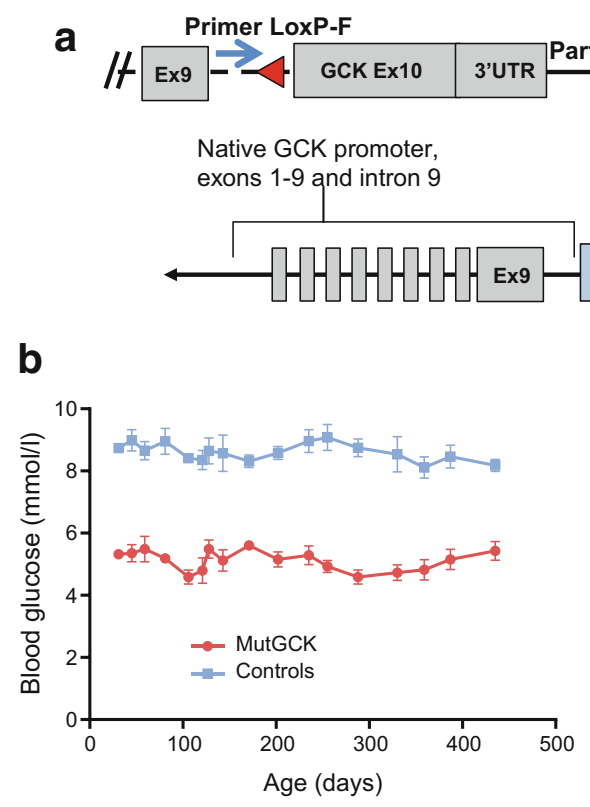

Primer LoxP-R

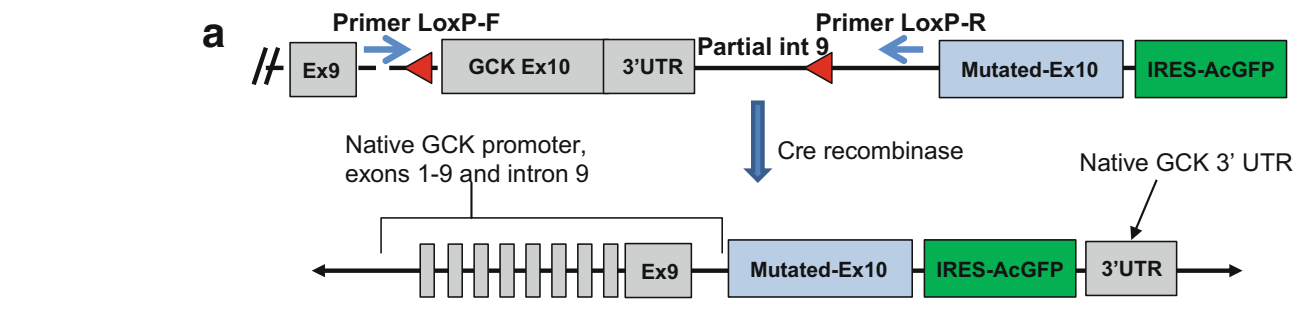

b
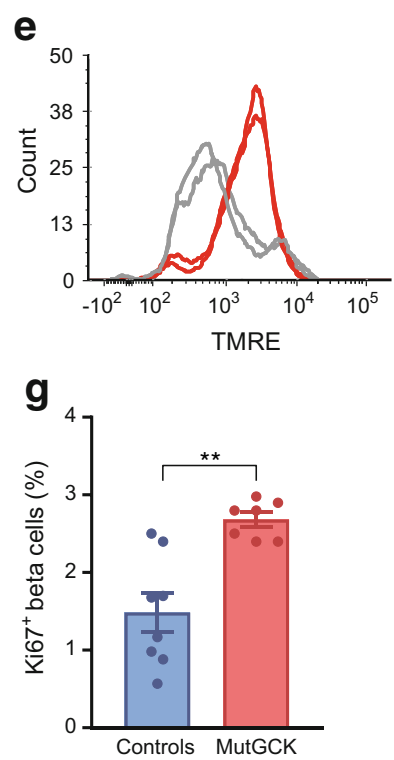

Fig. 1 Description and phenotype of novel model for GCK-CHI. (a) Schematic representation of mouse model. Upper panel, a cassette including the mutated Gck exon 10 followed by IRES-AcGFP was inserted immediately downstream to the native exon 10 , which was flanked by LoxP sites (red triangles). Thus, Cre recombinase results in removal of the native exon 10 and expression of mutant exon 10 in the natively regulated Gck locus, as shown in the lower panel. Two PCR primers (blue arrows, upper panel) were designed to measure efficiency of recombination. (b) Activation of mutGCK persistently decreased random blood glucose levels in beta-mutGCK mice (referred to as mutGCK in this and subsequent figures) ( $n=6$ mice/group). (c, d) Decreased glucose and increased insulin secretion in vivo after a $1 \mathrm{~h}$ fast in young mice $(n=9$ betamutGCK and 6 control mice). (e, f) FACS analysis of TMRE; beta cells

C

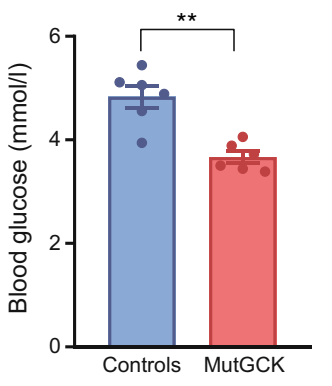

d

f

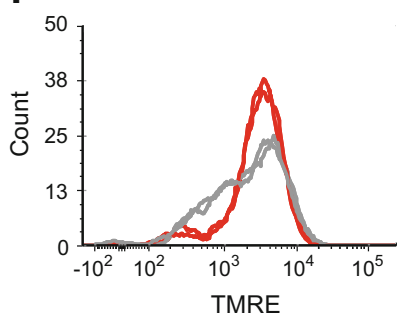

h

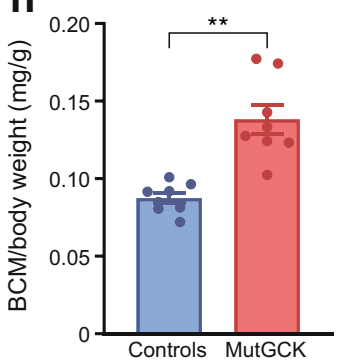

isolated from young control (e) and beta-mutGCK (f) mice. Grey lines represent islets incubated in $2.8 \mathrm{mmol} / 1$ glucose; red lines represent islets incubated in $16.7 \mathrm{mmol} / 1$ glucose. Islets from beta-mutGCK mice exhibited significantly increased membrane potential response in $2.8 \mathrm{mmol} / \mathrm{l}$ glucose compared with control mice ( $n=4$ mice/group). (g) The fraction of replicating beta cells is almost doubled in 1.5-month-old beta-mutGCK mice ( $n=8$ beta-mutGCK and 9 control mice). (h) Activation of betamutGCK caused an increase in beta cell mass as a function of body weight [(pancreas weight $\times \%$ beta cells)/body weight] at age 1.5 months ( $n=7$ beta-mutGCK and 8 control mice). Bars represent standard error $(* * p<0.001)$. BCM, beta cell mass; Ex, exon; int, intron; IRES-AcGFP, internal ribosome entry site followed by activated green fluorescent protein; UTR, untranslated region 
CreER) [17], as described in the Results and Discussion. In double transgenic offspring, beta cells are heterozygous for mutGCK, whose expression is regulated by native cis-regulatory elements, precisely mimicking the genetic status of GCKCHI. As no sexual dichotomy was observed, both male and female mice were used.

Animals were housed in the Hebrew University specific pathogen free (SPF) small animal facility and all studies were approved by and complied with the Animal Research Ethics Committees of the relevant institutions (the Hebrew University and the University of Pennsylvania).

Ex vivo perifusion assay For dynamic assessment of insulin secretion, islets were isolated from 1.5- and 8-month-old mutGCK and littermate control mice and loaded into a physiomimetic islet microsystem [18] installed in an automated perifusion system (Biorep Technologies, Florida, USA). Each well hosted 40 islets perifused at a flow rate of $100 \mu \mathrm{l} /$ $\mathrm{min}$ at $37^{\circ} \mathrm{C}$. Glucose was added to HEPES buffer $(125 \mathrm{mmol} /$ $1 \mathrm{NaCl}, 5.9 \mathrm{mmol} / \mathrm{l} \mathrm{KCl}, 2.56 \mathrm{mmol} / \mathrm{l} \mathrm{CaCl}, 1.2 \mathrm{mmol} / 1$ $\mathrm{MgCl}_{2}, 25 \mathrm{mmol} / \mathrm{l} \mathrm{HEPES}, 0.1 \% \mathrm{BSA}$, at $\mathrm{pH}$ 7.4) to achieve the desired concentrations. Depolarisation was induced by adding $25 \mathrm{mmol} / \mathrm{l} \mathrm{KCl}$. Perfusate was collected in 96-well plates and insulin was measured by ELISA (Crystal Chem no. 90082, IL, USA) and normalised to beta cell DNA content (DNeasy Blood and Tissue kit, Qiagen no. 51304, Hilden, Germany), based on assessing the methylation levels of a DNA region upstream of the transcription start site that is specifically unmethylated in beta cells and methylated in all other islet cell types, including alpha cells [19].

TMRE mitochondrial membrane potential analysis Dissociated islet cells were incubated with $7 \mu \mathrm{mol} / 1 \mathrm{TMRE}$ (tetramethylrhodamine, ethyl ester; Molecular Probes, OR, USA) for $1 \mathrm{~h}$ at $37^{\circ} \mathrm{C}$ in Krebs buffer containing 2.8 or $16.7 \mathrm{mmol} / 1$ glucose and measured by FACS.

Assessment of glucose/insulin tolerance and glucosestimulated insulin secretion GTTs and in vivo glucosestimulated insulin secretion (GSIS) assays were conducted as previously described [20]. Overnight-fasted mice received glucose by i.p. injection ( $2 \mathrm{mg} / \mathrm{kg}$ body weight), and blood glucose levels were measured as indicated. For insulin tolerance tests we pre-treated mice with $40 \mathrm{mg} / \mathrm{kg}$ diazoxide, a potent beta cell ATP-sensitive potassium channel $\left(\mathrm{K}_{\text {ATP }}\right.$ channel) opener, to suppress endogenous insulin secretion, and injected $10 \mathrm{U} / \mathrm{kg}$ insulin $1 \mathrm{~h}$ later. Blood glucose was measured as described [20].

Histology and immunostaining Tissue processing and immunostaining were performed as previously described [20] using antibodies listed in ESM Table 1. Fluorescent images were taken on a Nikon $\mathrm{C} 1$ confocal microscope (Tokyo, Japan) at $\times 40$ magnification. For beta cell mass determination, consecutive paraffin sections, $75 \mu \mathrm{m}$ apart, spanning the entire pancreas were stained for insulin and haematoxylin. Digital $\times 4$ images were obtained and stitched together using NISElements software (NIS-Elements Ar 3.22.00 [build 710] 3.22.0.7100. https://www.microscope.healthcare.nikon.com/ en_EU/products/software/nis-elements), and the fraction of tissue stained for insulin was determined.

Real-time PCR Islets were isolated from mutGCK and control mice. Total RNA was isolated, and cDNA synthesis was performed using a cDNA synthesis kit (Quanta Bioscience, MA, USA). For quantitative real-time PCR (qPCR), samples were run in triplicate using SYBR Green Master Mix (Quanta Bioscience) in the Bio-Rad 7900HT Fast Real-Time PCR System (Applied Biosystems, CA, USA). The relative amount of mRNA was calculated using the comparative $\mathrm{C}_{\mathrm{T}}$ method after normalisation to beta-actin. PCR primers are described in ESM Table 2.

\section{Results}

Short-term expression of mutGCK causes hyperinsulinaemic hypoglycaemia We employed a conditional gene replacement strategy (Fig. 1a, ESM Methods, ESM Figs. 1, 2) to engineer a mutant mouse strain in which Cre recombinase expression results in switching the wild-type (WT) exon 10 with a mutated exon 10 containing the ins $454 \mathrm{~A}$ activating mutation, without affecting native cis-acting regulatory mechanisms. This mutation, previously identified in a GCK-CHI patient, increases GCK affinity for glucose by sixfold, resulting in a decreased threshold for GSIS (0.8-1.4 mmol// vs $5 \mathrm{mmol} / \mathrm{l}$ of WT GCK) [14, 15]. Mice homozygous for the conditional mutant (mutGCK) were crossed with mice hemizygous for the rat insulin promoter-driven Cre recombinase (RIP-Cre) transgene [16], designated betamutGCK mice, or with mice heterozygous for an oestrogendependent Cre driven by the mouse insulin promoter (MIPCreER), designated cond-beta-mutGCK [17]. In all cases, mice were heterozygous for the mutant GCK allele. Heterozygous littermates lacking Cre recombinase were used as controls. Beta-mutGCK mice express the active mutant $G c k$ from the onset of insulin expression in fetal life ( gestational day 10.5), whereas cond-beta-mutGCK mice express the transgene following administration of tamoxifen. These mice were designed to express GFP after excision of the normal exon 10; however, although GFP mRNA expression was detected, GFP protein was not, probably due to failure of the internal ribosome entry site (IRES) cassette. To determine the efficiency of native exon 10 excision, we designed a PCR-based assay to detect the removal of the cassette between the two LoxP sites (ESM Fig. 3).

As expected, expression of the mutated Gck resulted in reduced random blood glucose levels $(4.5-5.4 \mathrm{mmol} / \mathrm{l})$, which persisted for at least 15 months, the maximum duration of the 
experiment (Fig. 1b). Consistent with the known function of GCK as the major glucose sensor in beta cells, overnightfasted glucose levels were lower (3.6 vs $4.8 \mathrm{mmol} / \mathrm{l}$, mutant and WT, respectively) and plasma insulin levels were higher compared with control mice $(0.25 \mathrm{vs} 0.10 \mathrm{pmol} / 1$, mutant and WT, respectively) (Fig. 1c,d).

Mitochondria respond to glucose stimulation by altering their membrane potential and ATP production [21, 22]. Using the mitochondrial dye TMRE, we determined that islets isolated from beta-mutGCK mice exhibited an increased membrane potential response at $2.8 \mathrm{mmol} / \mathrm{l}$ glucose compared with controls (Fig. 1e,f), as expected with this mutation.

\section{Activation of GCK causes increased beta cell proliferation and} mass in young mice In agreement with our previous reports [9, 13], beta cells of 1.5 -month-old beta-mutGCK mice exhibited approximately a twofold increase in cell cycle entry, quantified by immunostaining for the cell cycle marker Ki67 (2.7\% vs $1.5 \%$, mutant and WT, respectively, $p<0.01$; Fig. $1 \mathrm{~g}$ ), accompanied by a $62 \%$ increase in beta cell mass (Fig. 1h) but no difference in body weight, findings consistent with the increased islet size reported in infants with similar mutations [7]. Individual beta cell volume was measured in a subset of these animals and found to be unchanged, thus excluding hypertrophy as a mechanism for increased beta cell mass (ESM Fig. 4).

Long-term activation of GCK results in decreased glucose tolerance, increased DNA damage response and decreased beta cell mass Since mutGCK lowers blood glucose levels compared with WT GCK, we predicted that beta-mutGCK mice would display improved glucose tolerance. However, we detected no difference in GTTs in beta-mutGCK mice at 1.5 months of age compared with littermate controls (Fig. 2a). More surprisingly, at 8 months of age, these mice displayed impaired glucose tolerance (Fig. 2b), while maintaining fed and fasting hypoglycaemia. Several mechanisms could account for the observed glucose intolerance in older mice, including reduced beta cell function, decreased beta cell mass, increased insulin resistance or some combination thereof. To distinguish between reduced islet function and insulin resistance, we isolated islets from 1.5- and 8-month-old mice and performed a dynamic measurement of insulin secretion by perifusion (Fig. 2c,d). Since beta cell apoptosis has been reported in patients with activating Gck mutations [7] and in a mouse model expressing the Y214C GCK-activating mutation [13], we controlled for possible variability in beta cell numbers by normalising the concentration of secreted insulin to the number of beta cells in each assay using a beta cellspecific DNA methylation assay [19]. As shown in Fig. 2c,d, islets from beta-mutGCK mice secreted more insulin per beta cell following glucose stimulation compared with littermate controls at both ages, as expected from their lower basal glucose set point and demonstrating that beta-mutGCK beta cells maintain their functionality with age. This result indicates that the reduced glucose tolerance observed in older beta-mutGCK mice could not be explained by reduced cellautonomous beta cell function.

Next, we determined blood glucose levels in response to an exogenous bolus of insulin as a measure of peripheral insulin sensitivity. To prevent the confounding effect of counterregulatory hormone activation at very low glucose levels, we first treated the 8-month-old mice with diazoxide, a potent opener of the beta cell $\mathrm{K}_{\mathrm{ATP}}$ channels and suppressor of endogenous insulin secretion. Subsequent insulin administration induced a similar decrease in blood glucose levels $20 \mathrm{~min}$ post dose in beta-mutGCK and control mice (Fig. 2e). Although not the most sensitive test of peripheral insulin resistance, and acknowledging that off-target diazoxide effects cannot be excluded, these data suggest that it is unlikely that the glucose intolerance observed in older mutGCK mice is primarily driven by peripheral insulin resistance.

Finally, we evaluated beta cell mass of beta-mutGCK mice. We previously showed in the Y214C GCK-activating mutation mouse model that in addition to an acute mitogenic effect on beta cells, these mice also exhibit beta cell glucotoxicity in the form of DNA damage and cell death [13], consistent with observations of increased apoptosis in human GCK-CHI [7]. In the current model, the $62 \%$ increase in beta cell mass observed at 1.5 months relative to controls was completely reversed by 8 months of age, decreasing from $2.9 \mathrm{mg}$ at 1.5 months to $1.8 \mathrm{mg}$ at 8 months of age (Fig. $2 \mathrm{f}$ ). We hypothesised that this is most likely due to beta cell death, caused by oxidative and ER stress and DNA damage secondary to increased workload in the beta cells expressing the transgene. To determine whether the decrease in beta cell mass is constrained to beta cells expressing the mutated Gck, we evaluated the proportion of beta cells expressing Cre recombinase in islets from beta-mutGCK mice and found that the proportion of $\mathrm{Cre}^{+}$beta cells decreased from $71 \%$ in young mice to $48 \%$ in older mice (Fig. 3a,b). This was confirmed by measuring $\mathrm{Cre}^{+}$and $\mathrm{Cre}^{-}$beta cell mass separately in a subset of mice, observing that recombinant beta cell mass decreased from $2.9 \mathrm{mg}$ in young mice to $1.4 \mathrm{mg}$ in old mice whereas non-recombinant beta cell mass remained stable $(0.8$ vs $0.72 \mathrm{mg}$ in young and old mice, respectively) (Fig. 3c), suggesting that prolonged expression of the activated form of GCK has a slowly evolving, cell-autonomous glucotoxic effect on beta cells leading to loss of more than half of the mutant cells by 8 months of age.

Mechanism of beta cell damage after long-term activation of GCK We previously identified a molecular pathway of glucotoxicity, conserved from mouse to human, involving activation of the DNA double-strand break response [13]. To test whether this process is activated in the current model 
Fig. 2 Decreased glucose tolerance with age following activation of mutGCK. IPGTT: activation of beta-mutGCK has no effect on glucose tolerance at the age of 1.5 months (a) $(n=8$ beta-mutGCK and 9 control mice), but resulted in impaired glucose tolerance by the age of 8 months (b) ( $n=10$ betamutGCK and 9 control mice). Ex vivo dynamic measurements of insulin secretion, normalised to unmethylated DNA of the insulin 2 gene (perifusion): insulin secretion at age 1.5 months (c) and at 8 months of age (d) $(n=6$ mice/group). (e) Glucose response to i.p. insulin in mice pre-treated with diazoxide showing similar glucose clearance in WT and betamutGCK mice ( $n=8$ betamutGCK and 6 control mice). (f) Left, beta cell mass [(pancreas weight $\times \%$ beta cells)/body weight] in WT and mutant mice at the age of 1.5 months (young) ( $n=7$ mice in each group) and at 8 months of age (old) $(n=10$ mice in each group). $2.8 \mathrm{G}$, $2.8 \mathrm{mmol} / 1$ glucose; $16.8 \mathrm{G}$, $16.8 \mathrm{mmol} / 1$ glucose; $\mathrm{BCM}$, beta cell mass; unmeth, unmethylated. Bars represent standard error $(* p<0.05, * * p<0.001)$ a

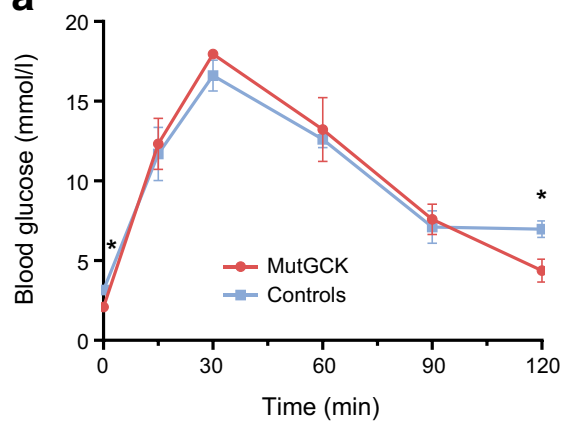

C

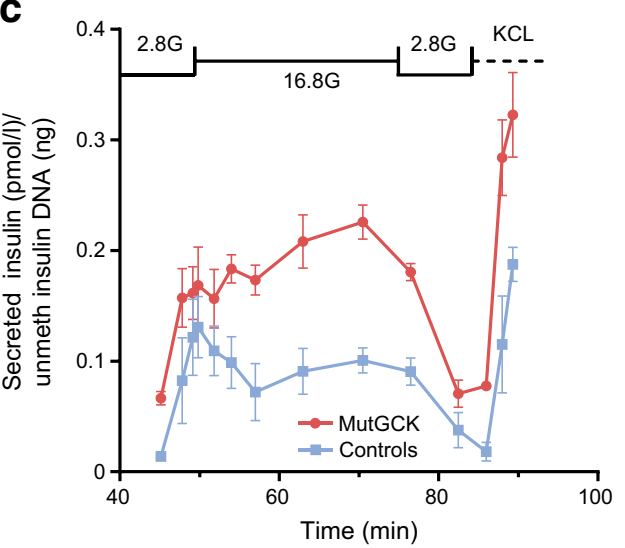

e

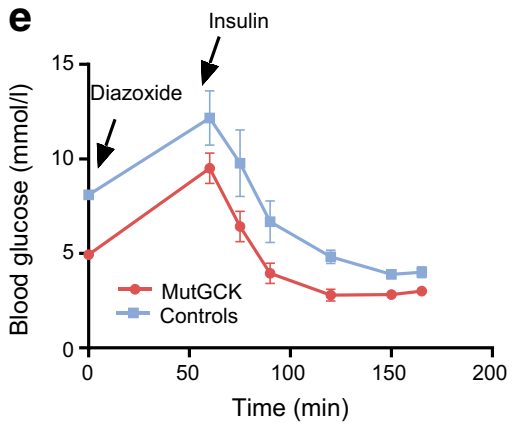

b

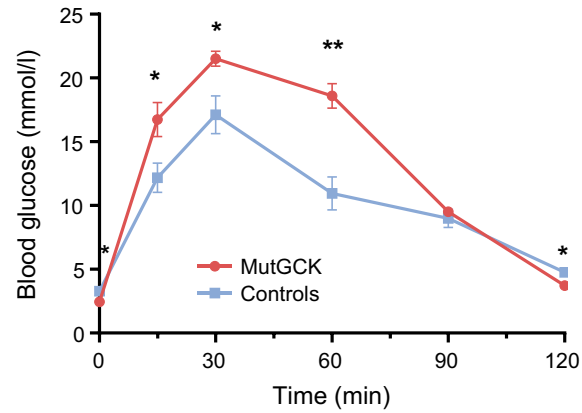

d
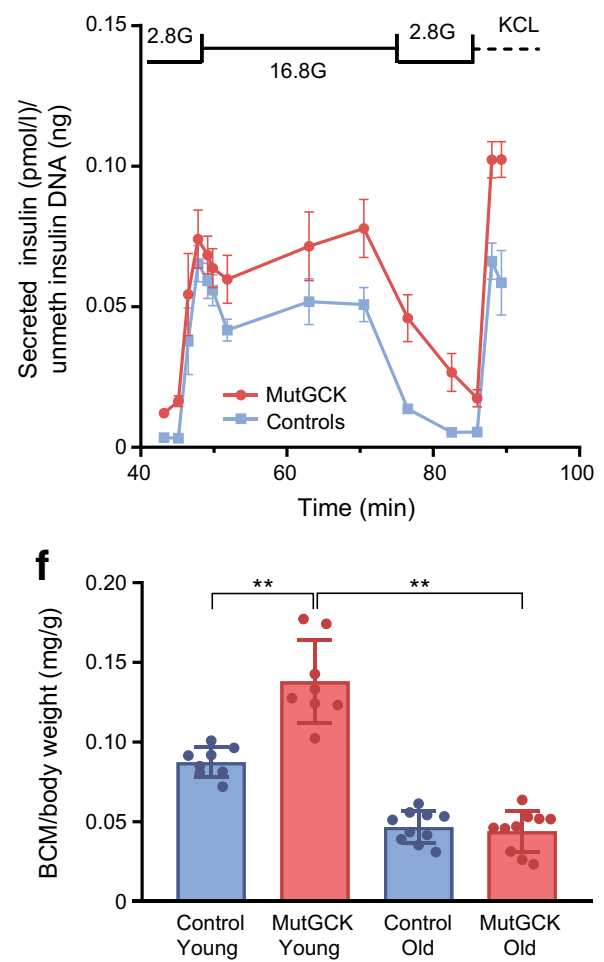

of prolonged exposure to activated GCK, we assessed the presence of tumour suppressor P53-binding protein 1 (TP53BP1) foci in young and old beta-mutGCK mice [23]. Notably, $4.5 \%$ of beta cell nuclei in young beta-mutGCK mice contained focal TP53BP1 staining compared with only $1.2 \%$ in controls, a finding that persisted in old mice (Fig. 3d).

To further characterise the basis of the apparent toxic effect of the mutGCK on beta cells, we examined the expression of the stress-related genes Chop (DNA damage-inducible transcript 3), Atf3 and spliced Xbp1, specific markers of ER stress [24]. qPCR analysis revealed a fourfold increase in the steadystate levels of Chop mRNA in islets of beta-mutGCK mice and a non-significant elevation in Atf3 and spliced $X b p 1$ levels (Fig. 3e).

Taken together, these findings suggest that, whereas during fetal and early postnatal life the activating mutation in $G c k$ enhances beta cell expansion, increased glycolytic flux and, thus, beta cell workload lead to cellular stress, DNA damage and cell death over time. This is consistent with previous data showing that low levels of ER and oxidative stress can have a pro-proliferative effect, while high levels or prolonged stress trigger apoptosis [25]. The observed elevation of Chop, a proapoptotic transcription factor, supports an ER stress-related mechanism contributing to loss of beta cell mass, as reported by Song and colleagues who demonstrated that Chop deletion prevents diabetes-related beta cell apoptosis in mice [26]. We were unable to identify apoptosis by TUNEL staining, probably related to the short duration of TUNEL positivity (2-3 h) [27] and the slow progression of beta cell loss ( $\sim 50 \%$ over 6.5 months).

Beta-mutGCK mice display reduced beta cell compensation during metabolic stress To study the effect of increased metabolic load on beta cells expressing mutGCK, control and beta- 
Fig. 3 Long-term effect of activation of mutGCK on beta cell survival and stress response. (a, b) Representative photomicrographs of Cre-positive beta cells in young ( 1.5 months) and old (8 months) mice (insulin, green; Cre, red; DAPI, blue). Scale bars, $20 \mu \mathrm{m}$. (c) Absolute beta cell mass (mg) was determined in four young (1.5 months) and four old (8 months) mutGCK mice and recombinant (Cre positive, magenta) and non-recombinant (Cre negative, grey) beta cells were counted separately, showing marked decrease in recombinant beta cell mass. (d) Percentage of 53BP1-positive beta cells in WT and beta-mutGCK $(n=6$ mice/group). (e) Atf3, Chop, and Xbp 1 mRNA expression in WT and mutGCK mice at 8 months age $(n=5 \mathrm{mice} /$ group $) ;{ }^{*} p<0.05$, $* * p<0.001$ a

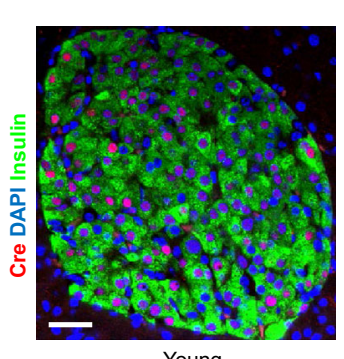

b

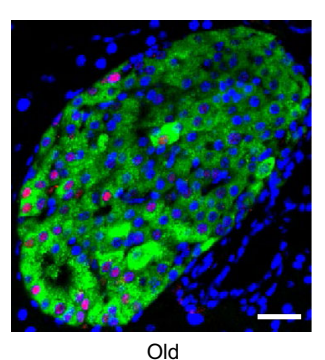

Old

d

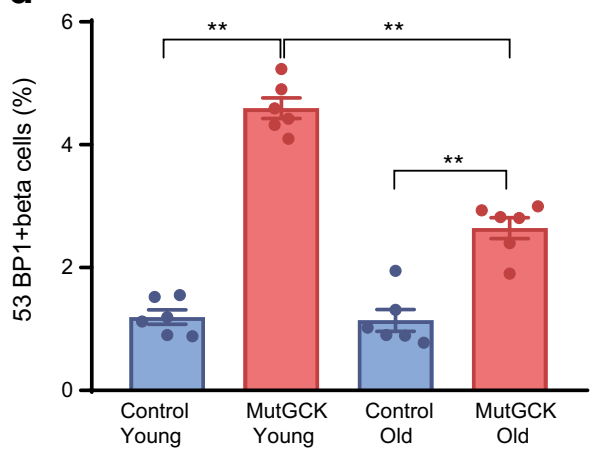

C

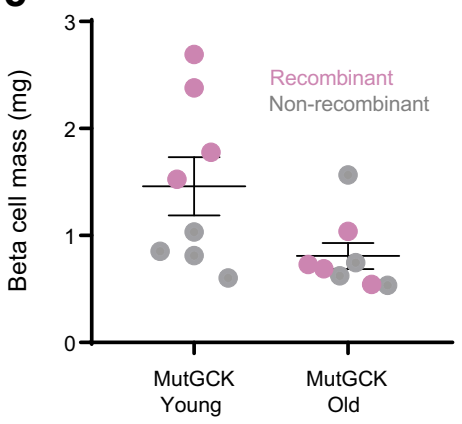

e

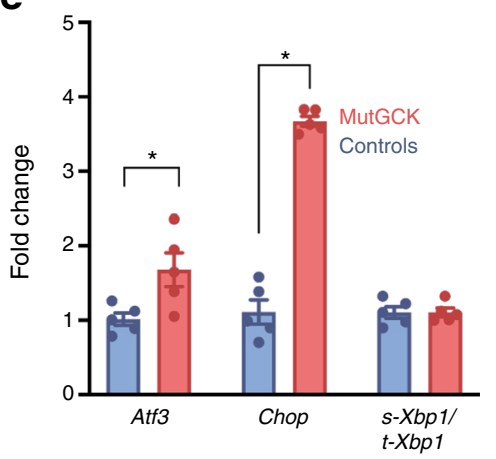

mutGCK mice were given a high-fat/high-sugar diet (HFD; $45 \%$ fat, $34 \%$ sucrose) for 37 weeks and assessed weekly for random blood glucose levels and body weight. GSIS, glucose tolerance and beta cell mass were measured at the beginning and end of the diet period. Whereas beta-mutGCK mice displayed consistently lower random glucose levels (Fig. 4a), body weight gain was similar in both groups (Fig. 4b). Chronic hyperinsulinaemia is expected to result in hyperphagia and increased body weight, which did not occur for reasons that could not be determined since caloric intake and energy expenditure were not measured. After 37 weeks of HFD, beta-mutGCK mice displayed more severely impaired glucose tolerance compared with WT mice (AUC 1796 vs $966 \mathrm{mmol} / \mathrm{l} \times \mathrm{min}$, mutant and WT, respectively, $p<0.05$ ) and mice maintained on normal laboratory chow (Fig. 4c,d). Interestingly, peak insulin levels $15 \mathrm{~min}$ following glucose injection, simulating first-phase insulin response, were slightly but significantly reduced in mutant mice compared with WT, despite identical blood glucose levels, suggesting a subtle defect in first-phase insulin response. Fasting glucose levels were lower and insulin levels were higher in the mutant mice, making the fold-change increase in insulin much reduced in mutant compared with WT mice $(1.7 \pm 0.4$ vs 6.2 $\pm 1.7, p<0.05$ ), a direct result of the Gck mutation and not evidence of dysfunction (Fig. 4e,f). Furthermore, while beta cell mass increased markedly in control mice as a result of HFD, the increase seen in beta-mutGCK mice was attenuated (Fig. 4g-i), demonstrating defective compensatory potential of beta cells expressing activated GCK.

\section{Discussion}

A faithful, conditional mouse model of GCK-CHI Here, we present a novel murine model that recapitulates human GCK-CHI and provides a likely mechanism for the progressive impairment of glucose tolerance observed in some patients with activating Gck mutations. This animal model constitutes a precise tool to study the effect of controlled, increased beta cell glycolysis without the confounding effects of ambient hyperglycaemia or hypoinsulinaemia, and thus addresses one potential mechanism of glucotoxicity relevant for type 2 diabetes progression. Unlike in our previous model [13], here, the conditional mutant gene is driven by the native GCK promoter and thus the phenotype observed is not confounded by unregulated expression driven by non-native cis-regulatory elements.

We used the Rip-Cre mouse to initiate recombination resulting in expression of the mutGCK during fetal development. Hypothalamic expression of both Rip-Cre and GCK has been reported $[28,29]$, potentially modifying the observed phenotype. However, only co-expression of both genes, as in the arcuate nucleus (ARC) and ventro-medial hypothalamus (VMH), will result in expression of recombinant Gck. Expression of mutant $G c k$ in the ARC is expected to affect appetite and weight, but our finding that beta-mutGCK and WT mice had similar weight throughout life suggests that the expression of mutGCK in this nucleus, if present, was of little or no physiologic consequence. Expression of mutGCK in the VMH is expected to affect counter-regulatory response to 
Fig. 4 Effect of HFD on betamutGCK mice. (a) Random (fed) glucose levels over a period of 40 weeks in control and HFD mice $(n=8$ beta-mutGCK and 5 controls). (b) Body mass measured weekly over 40 weeks in the HFD beta-mutGCK and control mice ( $n=14$ betamutGCK and 11 controls). (c) IPGTT in control (blue, $n=5$ ) and beta-mutGCK (red, $n=8$ ) mice after 40 weeks on HFD. Data for mice fed regular chow diet are given for comparison. (d) Area under the glucose response curve. (e, f) Overnight fasting plasma glucose and insulin concentrations after 40 weeks on HFD ( $n=8$ beta-mutGCK and 5 controls). (g) Beta cell mass [(pancreas weight $\times \%$ beta cells $) /$ body weight] of control and betamutGCK mice on regular laboratory chow (RD) or HFD. (h, i) Representative photomicrographs demonstrating higher beta cell mass after HFD in WT compared with betamutGCK mice. Scale bars, $200 \mu \mathrm{m}$ for both. BCM, beta cell mass; $\mathrm{RD}$, regular diet; $\mathrm{T} 0$, time 0 , before glucose injection; $\mathrm{T} 15$, 15 min after glucose injection. $* p<0.05, * * p<0.001$ a

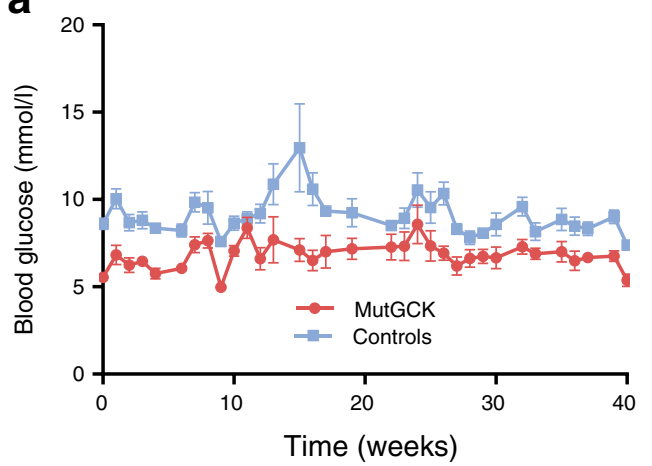

C

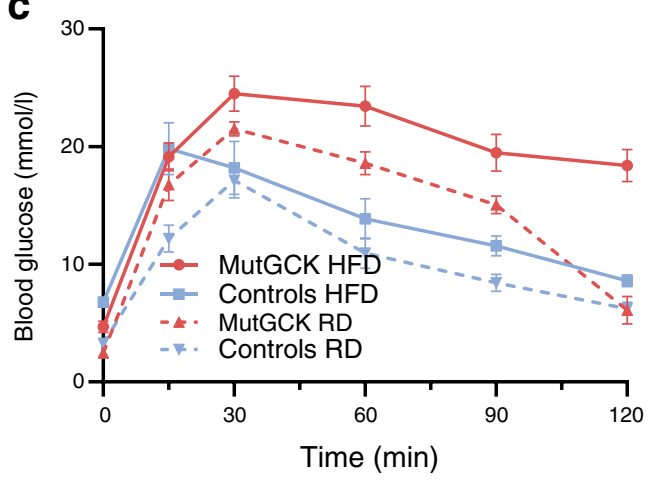

e

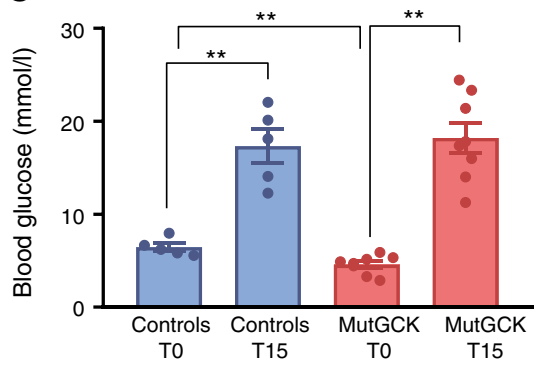

g

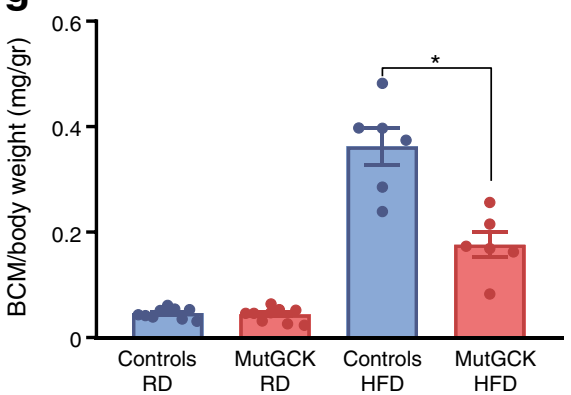

b

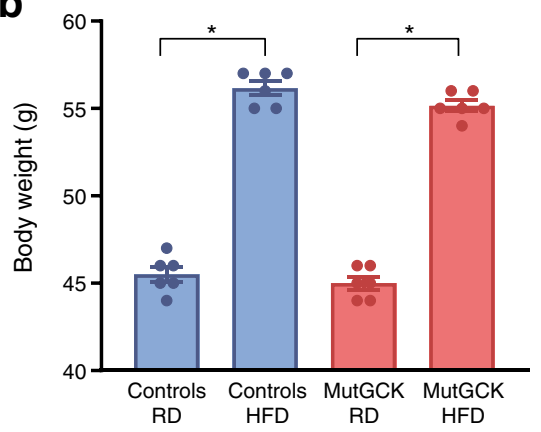

d
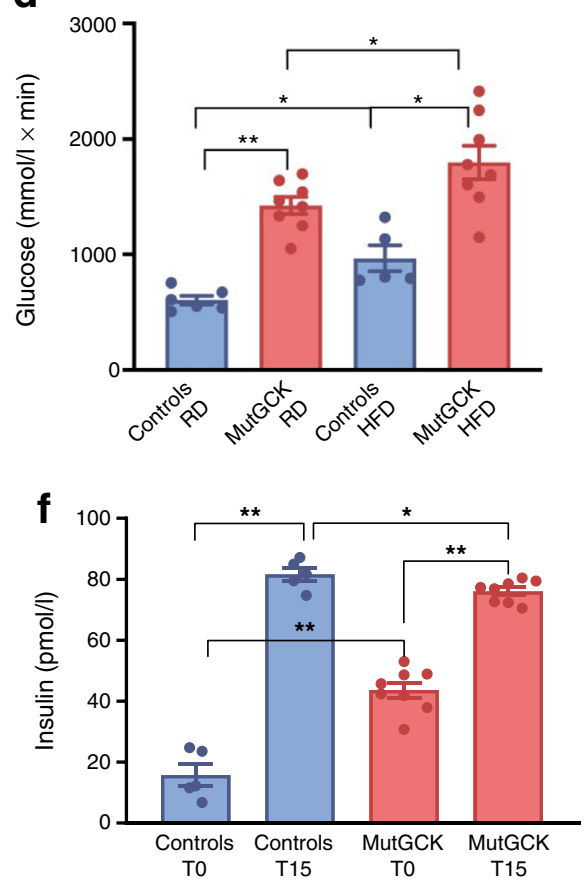

h
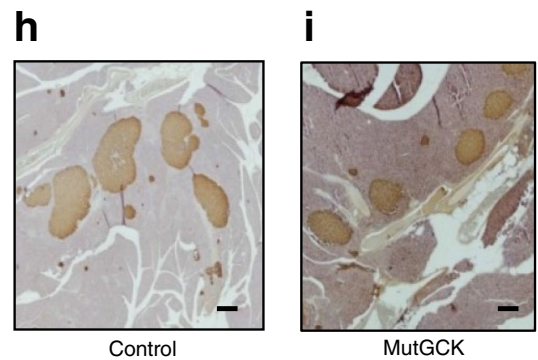

hypoglycaemia, but this is unlikely to directly affect beta cell proliferation or survival rate. To further confirm that hypothalamic Cre expression did not materially affect our results, we repeated key experiments using the MIP-CreER transgenic mouse and observed an essentially identical beta cell phenotype (ESM Figs. 5, 6).

We observed persistent fasting hypoglycaemia along with defective glucose response to i.p. glucose load in older animals. These two observations are not contradictory, as fasting glucose levels are determined by the glucose set point, dictated by GCK activity, and will be determined by remaining beta cells expressing the mutant Gck gene, which our perifusion experiments show maintain enhanced glucose responsiveness. In contrast, response to i.p. glucose infusion requires the maximum insulin secretory capacity of the full complement of beta cell mass. 
Biphasic dynamics of hyperglycolytic beta cells: mass expansion followed by contraction Increased metabolic demand and stress may cause beta cell compensation, followed in the long run by decompensation and beta cell failure, as observed clinically in people with type 2 diabetes [30] and in rodent models of diabetes (e.g., $d b / d b$ mice and Psammomys obesus under high-energy diet) [31]. A similar mechanism has been proposed in $\mathrm{CHI}$ caused by mutations in either of the $2 \mathrm{~K}_{\text {ATP }}$ channel genes $\left(\mathrm{K}_{\mathrm{ATP}}-\mathrm{CHI}\right)$, where severe hyperinsulinaemic hypoglycaemia tends to remit with time allowing increasing periods of fasting without hypoglycaemia [32], and which may further progress to glucose intolerance and even to diabetes. A similar clinical outcome, albeit milder and slower, has been proposed in some patients with GCK-CHI [13].

The molecular basis of beta cell failure in most of these scenarios remains unknown, with proposed mechanisms including pre-existing lower beta cell mass, failed beta cell compensation, dedifferentiation, dysfunction or cell death. In our study, the long-term deterioration of glycaemic control was not associated with reduced cell-autonomous beta cell functionality as determined by ex vivo perifusion (Fig. 2c,d) or reduced peripheral sensitivity to insulin (Fig. 2e). In contrast, we document an initial peak of beta cell proliferation and expansion of beta cell mass, followed by loss of hyperactive beta cells, ultimately leading to reduced beta cell mass.

\section{Mechanism of decreased beta cell mass following chronic hyperstimulation We demonstrated that in 8-month-old mice the mutant beta cell mass decreased by $\sim 52 \%$, while the mass of beta cells that did not express the mutant Gck was unchanged. This change was associated with evidence of an increased DNA damage response and ER stress. ER stress has been shown to be both beneficial and detrimental to beta cell function and survival [25]. Low levels of stress stimulate proliferation whereas high levels result in activation of the apoptosis cascade. Elevation of Chop is a marker of decom- pensated ER and oxidative stress, and indeed deletion of Chop has been shown to prevent beta cell death in various models of diabetes [26]. Therefore, our observation of increased expres- sion of Chop by activation of GCK provides a mechanism explaining decreased beta cell mass.}

Relevance to human disease We show how chronic overstimulation of beta cells drives a biphasic response: beta cell proliferation and mass expansion followed by reduced mass. We propose that the mechanisms responsible for this dynamic could contribute to type 2 diabetes development and suggest a unifying hypothesis addressing the long-standing controversy regarding the relative importance of beta cell mass vs function in the pathogenesis of type 2 diabetes [33-36].

We previously showed that beta cells respond acutely to increased workload by increasing proliferation [9]. Our new findings suggest that beta cells only tolerate increased workload transiently, eventually developing DNA damage and stress responses resulting in decreased survival rates. This cell-autonomous mechanism depends on the work required by the individual cell and not blood glucose levels per se, so that on the organismal level, individual beta cell workload is determined by both total beta cell number and whole-organism insulin requirement.

This 'workload hypothesis', which expands upon and modifies the mathematical model of type 2 diabetes pathogenesis proposed by Ha and colleagues [37], is supported by the observation that patients with MODY2, caused by heterozygous inactivating mutations in Gck, do not develop diabetes [38], unlike patients with similar fasting glucose levels due to impaired fasting glucose or glucose intolerance [30]. Despite similar fasting glucose levels, in MODY2, beta cell work is decreased, whereas in patients with impaired glucose tolerance the demand on the beta cell is increased, driven by increased extracellular glucose stimulating insulin secretion through normal GCK function.

Ex vivo perifusion studies did not identify any clear, cellautonomous beta cell dysfunction associated with the mutation. It is, however, highly likely that intrinsic beta cell dysfunction occurs over time, since beta cell stress and DNA damage result in loss of beta cell-specific gene expression, which could negatively influence overall islet function [39-41]. Indeed, the mild decrease in insulin levels $15 \mathrm{~min}$ after IPGTT in animals following HFD suggest a subtle defect in first-phase insulin response which must be confirmed by additional studies. Furthermore, our 'workload' hypothesis does not negate the possibility that hyperglycaemia or hyperlipidaemia per se could add another level of reversible or irreversible toxicity through multiple mechanisms, including protein or lipid glycation that could accelerate deterioration of beta cell function [42]. Even minor perturbations of fasting or stimulated blood glucose levels can contribute to progressive beta cell dysfunction, as recently shown in the rat $90 \%$ pancreatectomy model [43], although in this model beta cell workload is also increased.

Our findings have important implications for the prevention and treatment of type 2 diabetes, since they suggest that irreversible beta cell damage occurs as a function of workload, not related to elevated blood glucose levels per se. Genetic variation may play an important role in regulating peak beta cell mass and the ability of beta cells to cope with increased workload, but the impact of genetic variation is not yet sufficiently understood to permit targeted intervention. It is, however, possible to address non-genetic factors that affect peak beta cell mass, such as intrauterine and early childhood health, and those that affect whole-body insulin requirements, such as lifestyle choices. The data presented here suggest that to prevent type 2 diabetes, these factors should be addressed in early childhood and adolescence, long before the first evidence of glucose intolerance appears. 
Supplementary Information The online version contains peer-reviewed but unedited supplementary material available at https://doi.org/10.1007/ s00125-021-05390-x.

Acknowledgements The authors would like to thank all of the members of the Dor laboratory (Department of Developmental Biology and Cancer Research, Hebrew University-Hadassah Medical School, Jerusalem, Israel) for their helpful and constructive comments throughout the project; in particular, D. Neiman and R. Shemer for help in establishing the beta cell methylation assay and A. Klochendler for help in establishing additional assays used in this study.

Data availability Data, mouse models and genetic constructs generated and/or analysed during the current study are available from the corresponding author upon reasonable request.

Funding This study was supported by the National Institute of Diabetes and Digestive and Kidney Diseases (NIDDK) (UC4DK104119 to KHK and BG). DA was supported by NIDDK 5T32DK007314. BG was supported by the BIRAX Regenerative Medicine Initiative (14BX14NHBG), the Israel Science Foundation (ISF)-Juvenile Diabetes Research Foundation Joint Program in Type 1 Diabetes Research 1506/12, ISF grant 1782/18 and an EFSD award supported by the EFSD/JDRF/Lilly Programme in Type 1 Diabetes Research. YD was supported by grants from the DFG, the Ernest and Bonnie Beutler Research Program of Excellence in Genomic Medicine, the Alex U. Soyka pancreatic cancer fund, the Waldholtz/Pakula family and the Robert M. and Marilyn Sternberg Family Charitable Foundation. YD holds the Walter and Greta Stiel Chair and Research grant in heart studies. The study funders were not involved in the design of the study; the collection, analysis and interpretation of data; or the writing of the report; and did not impose any restrictions regarding the publication of the report.

Authors' relationships and activities The authors declare that there are no relationships or activities that might bias, or be perceived to bias, their work.

Contribution statement All co-authors made substantial contributions to conception and design, acquisition of data, or analysis and interpretation of data; were actively involved in drafting and critical revision of the manuscript; and approved the version of the manuscript to be published. NW-C derived the mouse model under the direction of KHK; ST-B performed the majority of the experiments and RSBH, JL and EF performed some experiments; and DA, KHK, YD and BG designed the experiments. ST-B drafted the manuscript. BG is the guarantor of this work and, as such, had full access to all of the data in the study and takes responsibility for the integrity of the data and the accuracy of the data analysis.

\section{References}

1. Back SH, Kaufman RJ (2012) Endoplasmic reticulum stress and type 2 diabetes. Annu Rev Biochem 81:767-793. https://doi.org/ 10.1146/annurev-biochem-072909-095555

2. Fonseca SG, Gromada J, Urano F (2011) Endoplasmic reticulum stress and pancreatic beta-cell death. Trends Endocrinol Metab 22(7):266-274. https://doi.org/10.1016/j.tem.2011.02.008

3. Kaneto H, Kajimoto Y, Fujitani Y et al (1999) Oxidative stress induces p21 expression in pancreatic islet cells: Possible implication in beta-cell dysfunction. Diabetologia 42(9):1093-1097. https://doi.org/10.1007/s001250051276
4. Robertson R, Zhou H, Zhang T, Harmon JS (2007) Chronic oxidative stress as a mechanism for glucose toxicity of the beta cell in type 2 diabetes. Cell Biochem Biophys 48(2-3):139-146. https:// doi.org/10.1007/s12013-007-0026-5

5. Robertson RP, Harmon J, Tran PO, Tanaka Y, Takahashi H (2003) Glucose toxicity in beta-cells: Type 2 diabetes, good radicals gone bad, and the glutathione connection. Diabetes 52(3):581-587. https://doi.org/10.2337/diabetes.52.3.581

6. Glaser B, Kesavan P, Heyman M et al (1998) Familial hyperinsulinism caused by an activating glucokinase mutation. N Engl J Med 338(4):226-230. https://doi.org/10.1056/NEJM199801223380404

7. Kassem S, Bhandari S, Rodriguez-Bada $P$ et al (2010) Large islets, beta-cell proliferation, and a glucokinase mutation. N Engl J Med 362(14):1348-1350. https://doi.org/10.1056/NEJMc0909845

8. Cuesta-Munoz AL, Huopio H, Otonkoski T et al (2004) Severe persistent hyperinsulinemic hypoglycemia due to a de novo glucokinase mutation. Diabetes 53(8):2164-2168. https://doi.org/10. 2337/diabetes.53.8.2164

9. Porat S, Weinberg-Corem N, Tornovsky-Babaey S et al (2011) Control of pancreatic beta cell regeneration by glucose metabolism. Cell Metab 13(4):440-449. https://doi.org/10.1016/j.cmet.2011.02. 012

10. Matschinsky FM, Porte D (2010) Glucokinase activators (GKAs) promise a new pharmacotherapy for diabetics. F1000 Med Rep 2: 43. https://doi.org/10.3410/M2-43

11. Meininger GE, Scott R, Alba M et al (2011) Effects of MK-0941, a novel glucokinase activator, on glycemic control in insulin-treated patients with type 2 diabetes. Diabetes Care 34(12):2560-2566. https://doi.org/10.2337/dc11-1200

12. Wilding JP, Leonsson-Zachrisson M, Wessman C, Johnsson E (2013) Dose-ranging study with the glucokinase activator AZD1656 in patients with type 2 diabetes mellitus on metformin. Diabetes Obes Metab 15(8):750-759. https://doi.org/10.1111/dom. 12088

13. Tornovsky-Babeay S, Dadon D, Ziv O et al (2014) Type 2 diabetes and congenital hyperinsulinism cause DNA double-strand breaks and p53 activity in beta cells. Cell Metab 19(1):109-121. https:// doi.org/10.1016/j.cmet.2013.11.007

14. Matschinsky FM (2009) Assessing the potential of glucokinase activators in diabetes therapy. Nat Rev Drug Discov 8(5):399 416. https://doi.org/10.1038/nrd2850

15. Sayed S, Langdon DR, Odili S et al (2009) Extremes of clinical and enzymatic phenotypes in children with hyperinsulinism caused by glucokinase activating mutations. Diabetes 58(6):1419-1427. https://doi.org/10.2337/db08-1792

16. Gannon M, Shiota C, Postic C, Wright CV, Magnuson M (2000) Analysis of the Cre-mediated recombination driven by rat insulin promoter in embryonic and adult mouse pancreas. Genesis 26(2): 139-142. https://doi.org/10.1002/(SICI)1526-968X(200002)26: $2<139::$ AID-GENE12>3.0.CO;2-7

17. Wicksteed B, Brissova M, Yan W et al (2010) Conditional gene targeting in mouse pancreatic ss-cells: Analysis of ectopic Cre transgene expression in the brain. Diabetes 59(12):3090-3098. https://doi.org/10.2337/db10-0624

18. Lenguito G, Chaimov D, Weitz JR et al (2017) Resealable, optically accessible, PDMS-free fluidic platform for ex vivo interrogation of pancreatic islets. Lab Chip 17(5):772-781. https://doi.org/10. 1039/c6lc01504b

19. Neiman D, Moss J, Hecht M et al (2017) Islet cells share promoter hypomethylation independently of expression, but exhibit celltype-specific methylation in enhancers. Proc Natl Acad Sci U S A 114(51):13525-13530. https://doi.org/10.1073/pnas.1713736114

20. Nir T, Melton DA, Dor Y (2007) Recovery from diabetes in mice by beta cell regeneration. J Clin Invest 117(9):2553-2561. https:// doi.org/10.1172/JCI32959 
21. Heart E, Corkey RF, Wikstrom JD, Shirihai OS, Corkey BE (2006) Glucose-dependent increase in mitochondrial membrane potential, but not cytoplasmic calcium, correlates with insulin secretion in single islet cells. Am J Physiol Endocrinol Metab 290(1):E143E148. https://doi.org/10.1152/ajpendo.00216.2005

22. Wikstrom JD, Katzman SM, Mohamed H et al (2007) Beta-cell mitochondria exhibit membrane potential heterogeneity that can be altered by stimulatory or toxic fuel levels. Diabetes 56(10): 2569-2578. https://doi.org/10.2337/db06-0757

23. Schultz LB, Chehab NH, Malikzay A, Halazonetis TD (2000) p53 binding protein 1 (53BP1) is an early participant in the cellular response to DNA double-strand breaks. J Cell Biol 151(7):13811390. https://doi.org/10.1083/jcb.151.7.1381

24. Cunha DA, Hekerman P, Ladriere L et al (2008) Initiation and execution of lipotoxic ER stress in pancreatic beta-cells. J Cell Sci 121(Pt 14):2308-2318. https://doi.org/10.1242/jcs.026062

25. Sharma RB, O'Donnell AC, Stamateris RE et al (2015) Insulin demand regulates beta cell number via the unfolded protein response. J Clin Invest 125(10):3831-3846. https://doi.org/10. 1172/JCI79264

26. Song B, Scheuner D, Ron D, Pennathur S, Kaufman RJ (2008) Chop deletion reduces oxidative stress, improves beta cell function, and promotes cell survival in multiple mouse models of diabetes. $\mathbf{J}$ Clin Invest 118(10):3378-3389. https://doi.org/10.1172/JCI34587

27. Elmore $S$ (2007) Apoptosis: A review of programmed cell death. Toxicol Pathol 35(4):495-516. https://doi.org/10.1080/ 01926230701320337

28. De Backer I, Hussain SS, Bloom SR, Gardiner JV (2016) Insights into the role of neuronal glucokinase. Am J Physiol Endocrinol Metab 311(1):E42-E55. https://doi.org/10.1152/ajpendo.00034. 2016

29. Song J, Xu Y, Hu X, Choi B, Tong Q (2010) Brain expression of Cre recombinase driven by pancreas-specific promoters. Genesis 48(11):628-634. https://doi.org/10.1002/dvg.20672

30. Kahn SE (2001) Clinical review 135: The importance of beta-cell failure in the development and progression of type 2 diabetes. J Clin Endocrinol Metab 86(9):4047-4058. https://doi.org/10.1210/jcem. 86.9.7713

31. Donath MY, Gross DJ, Cerasi E, Kaiser N (1999) Hyperglycemiainduced beta-cell apoptosis in pancreatic islets of Psammomys obesus during development of diabetes. Diabetes 48(4):738-744. https://doi.org/10.2337/diabetes.48.4.738

32. Glaser B, Landau H, Permutt MA (1999) Neonatal Hyperinsulinism. Trends Endocrinol Metab 10(2):55-61. https:// doi.org/10.1016/S1043-2760(98)00102-7

33. Holman RR, Clark A, Rorsman P (2020) Beta-cell secretory dysfunction: A key cause of type 2 diabetes. Lancet Diabetes Endocrinol 8(5):370. https://doi.org/10.1016/S2213-8587(20) 30119-4
34. Weir GC, Gaglia J, Bonner-Weir S (2020) Inadequate beta-cell mass is essential for the pathogenesis of type 2 diabetes. Lancet Diabetes Endocrinol 8(3):249-256. https://doi.org/10.1016/ S2213-8587(20)30022-X

35. Weir GC, Gaglia J, Bonner-Weir S (2020) Beta-cell secretory dysfunction: A key cause of type 2 diabetes - Authors' reply. Lancet Diabetes Endocrinol 8(5):370-371. https://doi.org/10. 1016/S2213-8587(20)30120-0

36. Butler AE, Janson J, Bonner-Weir S, Ritzel R, Rizza RA, Butler PC (2003) Beta-cell deficit and increased beta-cell apoptosis in humans with type 2 diabetes. Diabetes 52(1):102-110. https://doi.org/10. 2337/diabetes.52.1.102

37. Ha J, Satin LS, Sherman AS (2016) A mathematical model of the pathogenesis, prevention, and reversal of type 2 diabetes. Endocrinology 157(2):624-635. https://doi.org/10.1210/en.20151564

38. Martin D, Bellanne-Chantelot C, Deschamps I, Froguel P, Robert JJ, Velho G (2008) Long-term follow-up of oral glucose tolerance test-derived glucose tolerance and insulin secretion and insulin sensitivity indexes in subjects with glucokinase mutations (MODY2). Diabetes Care 31(7):1321-1323. https://doi.org/10. 2337/dc07-2017

39. Thompson PJ, Shah A, Ntranos V, Van Gool F, Atkinson M, Bhushan A (2019) Targeted elimination of senescent Beta cells prevents type 1 diabetes. CMET 29(5):1045-1060. https://doi.org/ 10.1016/j.cmet.2019.01.021

40. Aguayo-Mazzucato C, Andle J, Lee J, Terrence B et al (2019) Acceleration of $\beta$ cell aging determines diabetes and Senolysis improves disease outcomes. CMET 30(1):129-142.e124. https:// doi.org/10.1016/j.cmet.2019.05.006

41. Avrahami D, Wang YJ, Schug J et al (2020) Single-cell transcriptomics of human islet ontogeny defines the molecular basis of betacell dedifferentiation in T2D. Mol Metab 42:101057. https://doi. org/10.1016/j.molmet.2020.101057

42. Zhao Z, Zhao C, Zhang XH et al (2009) Advanced glycation end products inhibit glucose-stimulated insulin secretion through nitric oxide-dependent inhibition of cytochrome $\mathrm{c}$ oxidase and adenosine triphosphate synthesis. Endocrinology 150(6):2569-2576. https:// doi.org/10.1210/en.2008-1342

43. Ebrahimi AG, Hollister-Lock J, Sullivan BA, Tsuchida R, BonnerWeir S, Weir GC (2020) Beta cell identity changes with mild hyperglycemia: Implications for function, growth, and vulnerability. Mol Metab 35:100959. https://doi.org/10.1016/j.molmet.2020.02.002

Publisher's note Springer Nature remains neutral with regard to jurisdictional claims in published maps and institutional affiliations. 